\title{
Zeitschriften-Übersicht Journal Review Revue des Revues
}

The American Journal of Digestive Diseases, Vol. 5, No 5, May 1960

Auto-immune Reactions in the Course of Ulcer -ative Colitis. Polcak, J., M.D, and Vokurka, V., M.D.: Second Medical Clinic of the University Brno, Czechoslovakia.

Some Notes on the Epidemiology of Nonspecific Ulcerative Colitis. An Apparent Increase in Incidence in Jews. Weiner, H. A., M.D., and Lewis, Charles M., M.D.: Veterans Administration Hospital, East Orange, N.J.

The Effect of Certain Tranquilizers on Intra-bilîary Pressure of Cholecystectomized Dogs* Bianchi, R. G, B.S., and Craig, R. L, M.D., Ph.D.: Div. of Biological Research, G. D. Searle and Co., Chicago, 111.

The Use of Indocyanine Green for the Evaluation of Hepatic Function and Blood Flow in Man.

Wiegand, Bernard D. $\% \frac{5}{8}$ M.D.; Ketterer, Sig-

mund G., M.D., and Rapaport, Elliot, M.D.: Cardiopulmonary Laboratory, Mount Zion Hospital, San Francisco.

5. A Note on the Incidence of Pectoral Alopecia.

Gompertz, Michael L., M.D.: Veterans Administration Medical Teaching Group Hospital, Memphis 15, Tenn.

Intravenous Cholangiography with Sodium Iodopamidei The Problem of Nonvisualization. Floch, Martin H., M.D., and Geffen, Abraham, M.D,: Department of Radiology, Beth Israel Hospital, New York, N.Y.

Percutaneous Transhepatic Cholangiography. Kaplan, Allan A., M.D.; Brodsky, Leonard, M.D., and Rumball, John M., M.D.: Medical Service, Veterans Administration Hospital, Coral Gables, Fla.

Lactic Dehydrogenase Activity in Saliva, Bile, Gastric and Duodenal Contents. Levitan, R., M.D.; Golub, M., M.S., and Zetzel, L., M.D.: Beth Israel Hospital, Harvard Medical School; United States Public Health Service Trainee, Boston, Mass.

Plasma Pepsinogen and Adrenocortical Activity

New Data on the Relationship. Varro, V. M.D.; Faredin, I., Ph.D., and Cseraay, L., M.D.: Gastroenterological Research Laboratory, 1st Department od Medicine, University Medical School, Szeged, Hungary.

Carbonic Anhydrase Inhibition in the Management of Symptomatic Peptic Ulcers* Clinical Studies with Diamox on 125 Patients. Gailitis, Raimonds J., Major, M.C., and Schreiber, Werner, M.D.: Internal Medicine, University of Illinois, Chicago 3.

Calcifying Mucous-Cell Carcinoma of the Stomach $t$ Report of a Case. Khilnanî, Mansho T., M.B.: Department of Radiology, Mt. Sinai Hospital, New York 29, N.Y.

Homologous Serum Hepatitis Occurring in Laennec's Cirrhosis: Report of a Case. Wenger, Julius, M.D.; Turner, Daniel R., Capt., M.C., U.S.A., and Mendeloff, Joseph, M.D.: Medical Service and Laboratory Service of the Veterans Administration Hospital and the Emory University School of Medicine, Atlanta 19, Ga. 
Editorial $\mathrm{t}$ Diagnosis of Hiatal Hernia. Text or, E. Clinton, Jr., M.D., and Bundesen, William E., M.D.: Departments of Medicine and Radiology Northwestern University Medical Center, Chicago.

Zeitschriften-Übersicht - Journal Review - Revue des Revues 135

ad 1. Die Collodiumpartikel-Agglutination nach Cavelti mit autoptisch gewonnenem

Mucosaextrakt des Colon wurde mit 225 Patientenseren ausgeführt. Sie ergab aus-schließlich

positive Resultate bei 30 Patienten mit Colitis ulcerosa. Die Reaktion geht dem klinischen

Verlauf parallel und wird negativ nach totaler Colektomie, in einigen Fallen auch nach

eingetretener Heilung unter konservativer Therapie. Falls die Resultate der tschechischen

Autoren sich bestätigen, sind sie von weittragender Bedeutung, da sie die Rolle von

Autoimmunreaktionen in der Pathogenese der Colitis ulcerosa beweisen.

ad 2. Colitis ulcerosa scheint selten bei Negern, gehäuft bei Juden aufzutreten.

ad 3. Am cholezystektomierten Hund verursachen Chlorpromazin, Dartal und 3 weitere

Tranquilizers, i.v. verabreicht, innerhalb von 40-60 Minuten einen Druckanstieg in den

Gallenwegen.

ad 5. Allopezie der Brust bei 16\% weißer Manner und bei 46\% männlicher Neger. Keine

Häufung bei Leberzirrhose.

ad 6. Bei negativem i.v. Cholezystogramm wird regelmäßig eine Erkrankung der Leber oder der Gallenwege gefunden. Keine neuen Gesichtspunkte.

ad 7. Technik und Indikation der perkutanen transhepatischen Cholangiographie, die bei unklaren Fallen von Ikterus mit Hepatomegalie empfohlen wird. 8 eigene Fälle, von denen einer instruktiv in extenso beschrieben wird.

ad 8. Die Bestimmung der LDH im Magensaft ist für die Diagnose des Magenkarzi-noms nicht geeignet. Die Unterschiede gegenüber Ulcus sind nicht signifikant und die Fehlerquellen durch verschluckten Speichel oder Blutbeimengung groß.

ad 9. Beim Hund sinkt nach totaler Gastrektomie das Plasmapepsinogen auf mini-male Werte ab. ACTH i.v. stimuliert die Ausschüttung von Pepsinogen. Es kommt jedoch nicht zu einem Anstieg im peripheren Blut, da durch ACTH gleichzeitig die Nierenclearance gesteigert wird, was zu vermehrter Ausscheidung von Uropepsino-gen führt. Nach Adrenalektomie bleibt vermehrte Pepsinausschüttung unter Cinchopheneinwirkung aus.

ad 10. Diamoxtherapie bei Ulcuspatienten (1 $\mathrm{g}$ tgl.!) führt innerhalb von 5-14 Tagen zu einer Senkung der Magenazidität und einer Azidose. Das Urin-pH steigt initial von 6,0 auf 8,5, die Diurese von $\mathrm{Na}, \mathrm{K}$ und $\mathrm{Co} 2$ steigt an. Es kommt zu einer Ge-wichtsabnahme von mehreren Kilogramm und meist zu Inappetenz. Die Autoren führen den Rückgang der Ulcusbeschwerden ebenfalls auf die Diamoxwirkung zurück und glauben, es für die Ulcustherapie empfehlen zu dürfen, besonders zur Verhütung des Milch-Alkali-Syndroms.

ad 11. 12. Publizierter Fall der Literatur.

ad 12. Bioptisch verfolgter letaler Fall von portaler Zirrhose mit aufgepfropfter Serum-Hepatitis. ad 13. Kritische Stellungnahme zur Abgrenzung kleiner Hiatushernien von der

Norm mit interessanten cesophago-manometrischen Angaben. E. Hafter

Gastroenterology, Vol. 38, No. 5, Mai 1960

Hyperaldosteronism in Liver Disease. Henley, K. S.; Streeten, D. H. P. and Pollard, H. M.:

Depart, of Internal Medicine, Univ. of Michigan Med. School, Ann Arbor, Mich., p. 681-689.

Application of the Fluorescein String Test in 32 Cases of Upper Gastrointestinal Hemorrhage.

Haynes, W. F. Jr. and Pittmaa, F. E.: Depart, of Medicine, St. Luke's Hosp., New York, N. Y., p. 690-697. 
Serum Leucine Aminopeptidase in Pancreatic and Hepatobiliary Diseases. Pineda, E. P.; Goldbarg, J. A.; Banks, B. M. and Rutenburg, A. M.: Depart, of Surgery, Beth Israel Hosp. and Harvard Med. School, Boston, Mass., p. 698-712.

A New Qualitative Defect of Leukocyte Function in Ulcerative Colitis. Priest, R. J.; Rebuck, J. W. and Havey, G. T.: Div. of Gastroenterology and Depart, of Labor., Henry Ford Hosp., Detroit, Mich., p. 715-720.

Segmental (Ulcerative) Colitis. Yarnis, H. and Crohn, B. B.: Mt. Sinai Hosp., New York, N. Y., p. $721-728$.

Effects of Glucagon and Insulin on Gastric Secretion in Rats. Jow, E.; Webster, D. R. and

Skoryna S. C.: Gastro-Intestinal Research Lab. and Depart, of Experim. Surgery, McGill Univ., Montreal, Canada, p. 732-739.

136 Zeitschriften-Übersicht - Journal Review - Revue des Revues

7. Effect of Small and Massive Doses of Prednisone 14. American Association for the Study of Liver on Gastric Secretory Activity. Beck, I. T.; Diseases. Short Presentations, p. 792-804.

Fletcher, H. W.; McKenna, R. D. and Griff, H.:

University Clinic, McGill Univ., and Depart, of 15. Granular Cell Myoblastoma of the Esophagus.

Medicine, Gastroenterology, Royal Victoria De Gouveia O. F.; Pereira A.A.; Netto M. B.; Hosp., Montreal, Canada, p. 740-749 Vilhena, A. M.; Dutra, G. and Bryk, D.: National Faculty of Med., Univ. of Brazil, Rio de Janeiro,

8. Parathyroids and Gastric Secretion. Donegan, W. Brazil, p. 805-809.

L. and Spiro, H. M.: Depart, of Internal Medicine, Yale Univ. School of Med., New Haven, $\quad$ 16. Primary Carcinoma of the Jejunum with Atrophic

Conn d 750-759 Jejunitis and Intestinal Malabsorption. Joske,

“، R. A.: Depart, of Med., Royal Perth Hosp. and

9. Follow-up Study of 127 Patients with Stomal Univ of Western Australia, Perth, Australia, $\mathrm{p}$.

Ulcer. Boles, R. S., Jr.; Marshall, S. F. and 810-816.

Bersoux RV.rDepart ofGasWoenterologyand 17. Carcinollla in , L Pharyngoesophageal Surgery, Lahey Chn,c, Boston, Mass., p. 763-766. Díverticulum. Liberson, M. and Riese, K. T.:

$\Lambda \ldots \ldots \ldots, \ldots \quad$ Depart, of Radiology and Surgery. State Vet.

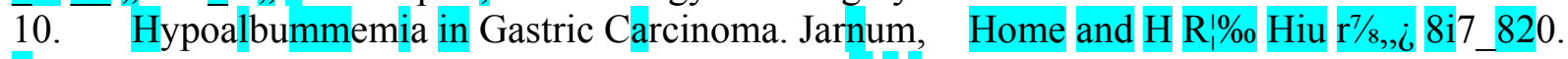
b. and Schwartz, M.: Surgical Depart. A, Med. $\quad$ Г J r Depart. B, Depart, of Clin. Biochemistry andı8. Chronic Granulomatous Cholecystitis and Chronic

Physiology, Bispebjerg Hosp., Copenhagen, Fibrosing Choledochitis Associated with Chronic Denmark, p. 769-776. Ulcerative Colitis. Goldgraber, M. B. and Kirsner,

J. B.: Depart, of Medicine, Univ. of Chicago, 111.

11. Clinical Evaluation of the Effectiveness and p 321-828.

Safety of Trimethobenzamide (Tigan). Brandman, 0.: HarrisonS. Martland Med. Center and Health 19. Chronic Hvperbilirubinemia with Normal Liver 
Depart, of Newark, N. J., p. 777-780. Biopsy. Willner, V.; Treiber, M. W. and Manderson, L. V.: Medical Service, Meadowbrook Hosp.,

12. Mortality Factors in Experimental Hemorrhagic Hempstead, N. Y., p. 829-831. Pancreatitis. Ulin, A. W. and Sokolic, I. H.: S. B. Mandell Lab. of Research Surgery, A. Einstein the Abdo-

Med. Center, and Hahnemann Med. School and

20. Editorial: Remote Pain from Disease in Hosp., Philadelphia, Pa., p. 781-785.

21. Distinctive Elcctrophoretic Pattern of Gastric

13. Symposium on Toxic Hepatic Injury. Popper, H., Juice in Pernicious Anemia and Achlorhydria

Symposium on Toxic Hepatic Injury. P

Moderator, p. 786-792. Glass, G. B. J., New York, N. Y., p. 837-840.

ad 1. II est probable que $\Gamma$ hyperaldosteronisme joue un role dans la pathogenèse de la retention hydrique et de $\Gamma$ ascite au cours des cirrhoses. Ce role est illustré par 4 cas; un malade n'a vide son ascite qu'après surrénalectomie bilatérale et les 3 autres grace à $\Gamma$ administration d'antagonistes de $\Gamma$ aldostérone (spirolactones), combines au chlorothiazide.

ad 2. Afin de situer rapidement Torigine d'une hémorragie gastro-duodénale, on emploie un cordon qu'on fait avaler au patient tandis qu'on lui injecte de la fluo-rescéine i.v. On retire le cordon après 4 minutes et on recherche les traces de sang et la fluorescence aux rayons ultraviolets dans $\Gamma$ obscurité.

ad 3. La LAP (leucine aminopeptidase) est une enzyme protéolytique surtout représentée dans le foie, pancreas, rein et intestin grêle. En cas de cancer de la tête du pancreas ou des voies biliaires extra-hépatiques, son taux est très élevé. II est plus haut en cas d'obstruction tumorale qu'en cas de calcul. Dans Tobstruction cholédocienne, la LAP s'élève souvent avant la bilirubine ou la phosphatase alcaline. Augmentation fréquente aussi dans le cancer de la queue et du corps du pancreas, plus faible dans les hepatites toxiques et à virus; par contre, LAP reste plus long-temps perturbée que les autres tests hépatiques. Les métastases hépatiques élèvent la LAP même en Tabsence d'ictère.

ad 5. Un grand nombre de côlites segmentaires sont régionales et souvent situées à droite; dans beaucoup de cas, les lesions sont aussi granulomateuses qu'ulcéreuses. Les cas limités au colon droit n'évoluent pas souvent du côté distal.

ad 7. Chez le chien ou chez l'homme, que ce soit avec de faibles ou de fortes doses de prednisone, la secretion gastrique est peu modifiée. Si done ce stéroïde a une action ulcérigène, ce n'est pas par l'effet d'une hypersécrétion.

ad 8. L'injection de parathormone augmente la secretion gastrique. L'hypoparathyroïdisme s'accompagne d'hyposécrétion gastrique, qui est corrigée lorsque la calcémie est normalisée, même en Tabsence de Thormone manquante. L'ion calcique parait nécessaire à la fonction des glandes gastriques.

B. Wíssmer, Geneve 